\title{
EQUILIBRIUM POSITIONS FOR EQUALLY CHARGED PARTICLES ON A SURFACE ${ }^{1}$
}

\author{
BY JAMES H. WHITE
}

Communicated by S. S. Chern, November 9,1972

\begin{abstract}
This paper gives a lower bound for the number of equilibrium positions of two or three equally charged particles on an imbedded surface in Euclidean $n$-space.
\end{abstract}

Let $f: M \rightarrow E^{n}$ be a $C^{k}(k \geqq 2)$ imbedding of a closed orientable surface into Euclidean $n$-space which is generic in a certain sense. This paper announces results on the lower bounds for the number of equilibrium positions of two or three equally charged particles on $f(M)$ and indicates, thereby, the manner in which the general case can be studied. For simplicity all charges are assumed to be +1 .

1. The 2 particle case. The imbedding $f: M \rightarrow E^{n}$ is said to be $V$-generic (potential-generic) if the function $V_{f}: M \times M-D \rightarrow \mathrm{R}$ defined on $M \times M$ outside of the diagonal $D$ by

$$
V_{f}(x, y)=1 /\|f(x)-f(y)\|
$$

satisfies the property that on $M \times M-D$ all its critical points are nondegenerate. (Any $C^{k}(k \geqq 2)$ imbedding of $M$ satisfies the property that there exists a real number $N$ such that, if $V_{f}(x, y) \geqq N,(x, y)$ cannot be a critical point of $V_{f}$.)

$V_{f}$ can be easily recognized to be the potential of two unit charges on $f(M)$, so that the critical points of $V_{f}$ are in fact the equilibrium positions. To compute the lower bound for the number of such positions, one observes that on $M \times M-D$, the critical points of $V_{f}$ are the same as those of the function $V_{f}^{-2}$, that is, the function which assigns to $(x, y)$ the number $\|f(x)-f(y)\|^{2}$. One may then apply the work of $[1]$ to obtain

THEOREM 1. Let $f: M \rightarrow E^{n}$ be a V-generic imbedding of a surface of genus $g$ into $E^{n}$. Then the lower bound for the number of equilibrium positions of two equally charged particles on $f(M)$ is $2 g^{2}+3 g+3$.

2. The 3 particle case. The 3 particle case is exceedingly more difficult because of the homology theory involved and thereby gives an indication of the difficulty of the general case.

Consider the triple cartesian product of $M$ with itself, $M \times M \times M$,

AMS (MOS) subject classifications (1970). Primary 58E05; Secondary 70F10.

Key words and phrases. Equilibrium positions, charged particles, Morse theory, potential, lower bound.

${ }^{1}$ Preparation of this paper was supported in part by NSF Grant No. GP-27576. 
and let $A$ be the total diagonal, i.e.

$$
A=\{(x, y, z) \in M \times M \times M \mid x=y \text { or } x=z \text { or } y=z\} .
$$

The imbedding $f: M \rightarrow E^{n}$ is said to be $V$-generic if the function $V_{f}: M \times M \times M-A \rightarrow R$ defined by

$$
V_{f}(x, y, z)=\frac{1}{\|f(x)-f(y)\|}+\frac{1}{\|f(y)-f(z)\|}+\frac{1}{\|f(z)-f(x)\|}
$$

satisfies the property that on $M \times M \times M-A$ all its critical points are nondegenerate. $V_{f}$ is the potential function for three equally charged particles and its critical points are the equilibrium positions.

It can be shown that there exists a number $N$ such that, if $V_{f}(x, y, z) \geqq N$, $(x, y, z)$ cannot be a critical point of $V_{f}$. Let

$$
A_{N}=\left\{(x, y, z) \in M \times M \times M \mid V_{f}(x, y, z)>N\right\} .
$$

To compute the lower bound for the equilibrium positions, one applies Morse theory to the function $V_{f}$ on the set $M \times M \times M-A_{N}$. One finds that the number of critical points of $V_{f}$ depends on the Betti numbers of the pair $(M \times M \times M, A)$.

To each equilibrium position of $V_{f}$, there corresponds six critical points of $V_{f}$ for if $(x, y, z)$ is a critical point, then so is any triple which is a permutation of $x, y$, and $z$. The index of an equilibrium position is defined to be the index of the corresponding critical point, so that if $c_{i}$ is the number of equilibrium positions of index $i, V_{f}$ has $6 c_{i}$ critical points of index $i$. The theorem may be stated as follows:

THEOREM 2. Let $b_{i}$ be the ith Betti number of $(M \times M \times M, A)$ and let $c_{i}$ be the number of equilibrium positions of index $i$. Then

$$
6 \sum_{j=0}^{i}(-1)^{j} c_{i-j} \geqq \sum_{j=0}^{i}(-1)^{j} b_{i-j}, \quad i=0, \ldots, 6 .
$$

COROLlaRY. The lower bound for the number of equilibrium positions is

$$
2 \sum_{i=0}^{6} \sum_{j=0}^{i}\left[\frac{(-1)^{j} b_{i-j}}{6}\right]-\left[\sum_{j=0}^{6} \frac{(-1)^{j} b_{6-j}}{6}\right],
$$

where $[\kappa / 6]$ is the smallest integer $\geqq \kappa / 6$.

To compute the Betti numbers of $(M \times M \times M, A)$ is rather difficult. The outline of this computation is as follows. First, one easily computes the Betti numbers of $M \times M \times M$. Next one uses Mayer-Vietoris sequences to compute the Betti numbers of $A$, observing the fact that $A$ is essentially three copies of $M \times M$ joined along a single copy of $M$. One next calls on the relative exact sequence $\cdots \rightarrow H_{*}(A) \stackrel{i_{*}}{\rightarrow} H_{*}(M \times$ 
$M \times M) \rightarrow H_{*}(M \times M \times M, A) \rightarrow \cdots$ to compute the Betti numbers of $(M \times M \times M, A)$, where $i_{*}$ is the induced map from the inclusion $i: A \rightarrow M \times M \times M$. However, to determine the kernel or image of $i_{*}$ is by no means an easy task, since this map is not always one-to-one or onto. One proceeds as follows. Let $d: M \rightarrow M \times M$ denote the diagonal map, i.e. $d(x)=(x, x)$. Define three maps

$$
j_{\alpha}: M \times M \rightarrow M \times M \times M, \quad \alpha=1,2,3,
$$

by

$$
\begin{aligned}
& j_{1}(x, y)=(d \times \mathrm{id})(x, y)=(x, x, y) \\
& j_{2}(x, y)=(\mathrm{id} \times \mathrm{tw}) \circ(d \times \mathrm{id})(x, y)=(x, y, x) \\
& j_{3}(x, y)=(\mathrm{id} \times d)(x, y)=(x, y, y),
\end{aligned}
$$

where id is the identity $\operatorname{map}, \operatorname{id}(x)=x$, and tw is the twist map, $\operatorname{tw}(x, y)=$ $(y, x)$. Since the diagrams

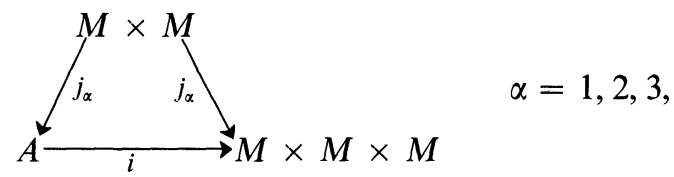

commute, the following diagram commutes:

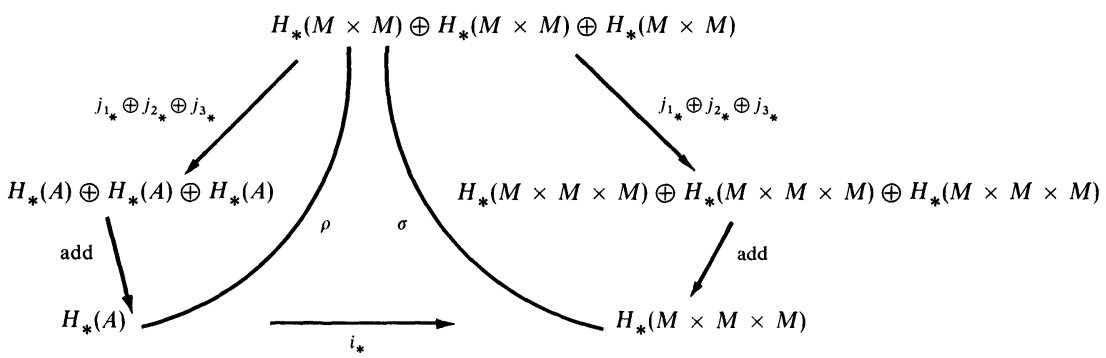

where add is just the simple addition in $H_{*}(A)$ and $H_{*}(M \times M \times M)$ respectively of the images of $j_{1_{*}}, j_{2 *}$, and $j_{3 *}$ in $H_{*}(A)$ and $H_{*}(M \times M \times M)$ respectively, and where $\rho$ and $\sigma$ are the composition maps add $\circ j_{1} \oplus$ $j_{2 *} \oplus j_{3 *}$.

It is not too difficult a task to determine the kernel and image of $\rho$; in fact, it is always either an isomorphism or onto. It is, however, quite difficult to determine the image and kernel of $\sigma$, but with patience it may be done quite directly since the ring cohomology structure for surfaces is known. Once $\rho$ and $\sigma$ are completely known one can determine the kernel and image of $i_{*}$.

Finally, if one gathers all the information together one obtains that, 
except for the torus, the Betti numbers of $(M \times M \times M, A)$ are $b_{0}=0$, $b_{1}=0, b_{2}=2 g^{2}+4 g, b_{3}=8 g^{3}+2 g^{2}+2 g+1, b_{4}=12 g^{2}, b_{5}=6 g$, $b_{6}=1$.

Using the corollary we obtain

THEOREM 3. Let $f: M \rightarrow E^{n}$ be a V-generic imbedding of a surface of genus $g \neq 1$ into $E^{n}$. Then the lower bound for the number of equilibrium positions of three equally charged particles on $f(M)$ is

(a) $\left(4 g^{3}+8 g^{2}+6 g+12\right) / 3 g \neq 2(\bmod 3)$,

(b) $\left(4 g^{3}+8 g^{2}+6 g+14\right) / 3, g \equiv 2(\bmod 3)$.

For the torus special considerations must be made and the lower bound is eleven.

REMARK. The case of three charged particles on a curve in $E^{n}$ is easily done and the lower bound is found to be two.

The author gratefully acknowledges the valuable assistance of Professor Robert Brown in the homology theory of this work.

\section{REFERENCES}

1. F. Takens and J. White, Morse theory of double normals of immersions, Indiana $\mathrm{J}$. Math. 21 (1971), 11-17.

Department of Mathematics, University of California, Los Angeles, California 90024 